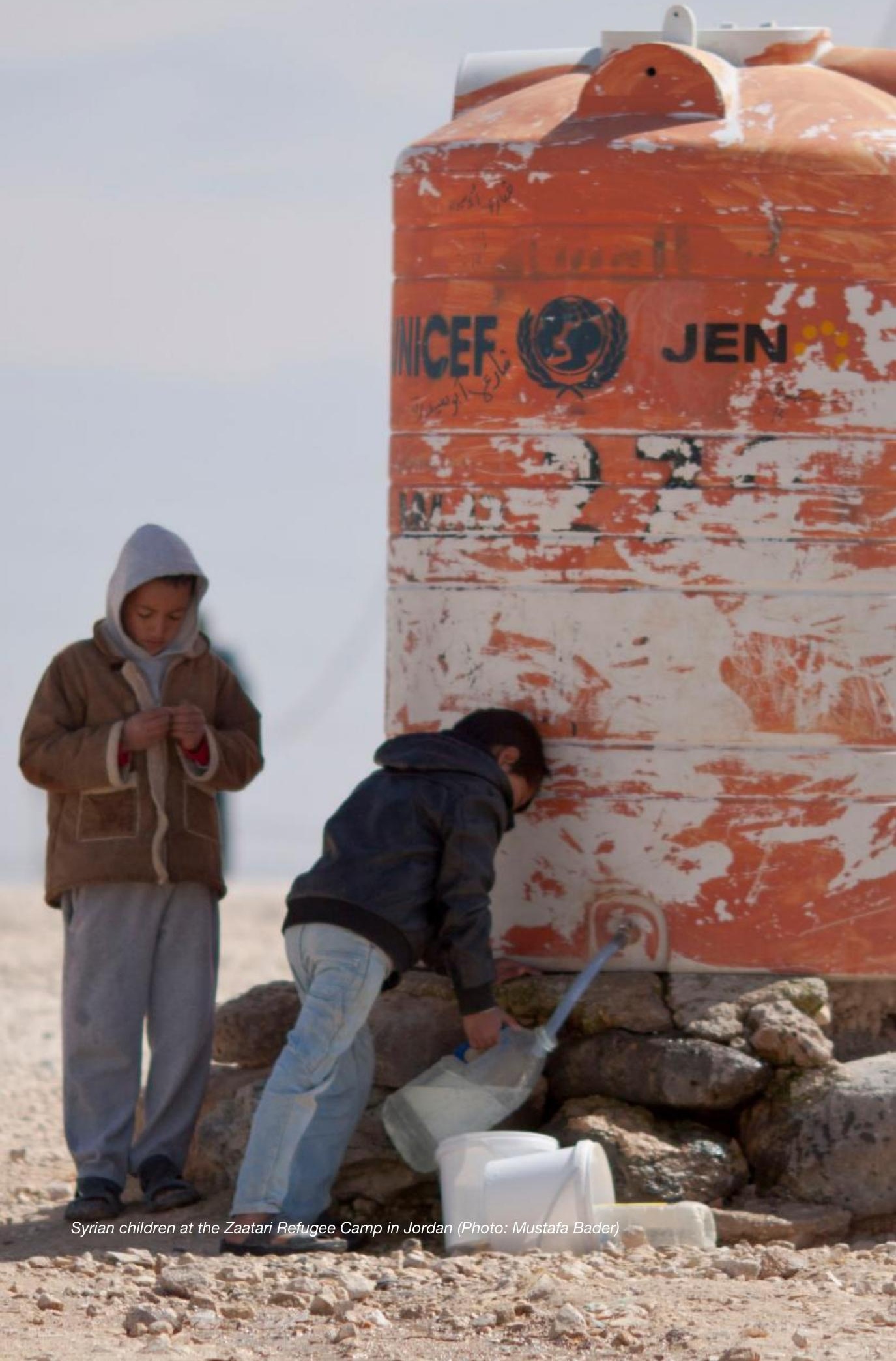




\section{COMMENTARY: SYRIAN REFUGEE CHILDREN AND MENTAL HEALTH TRAUMA}

By Dr. Rania Abuelhassan

Gender-based violence program specialist,

Damascus

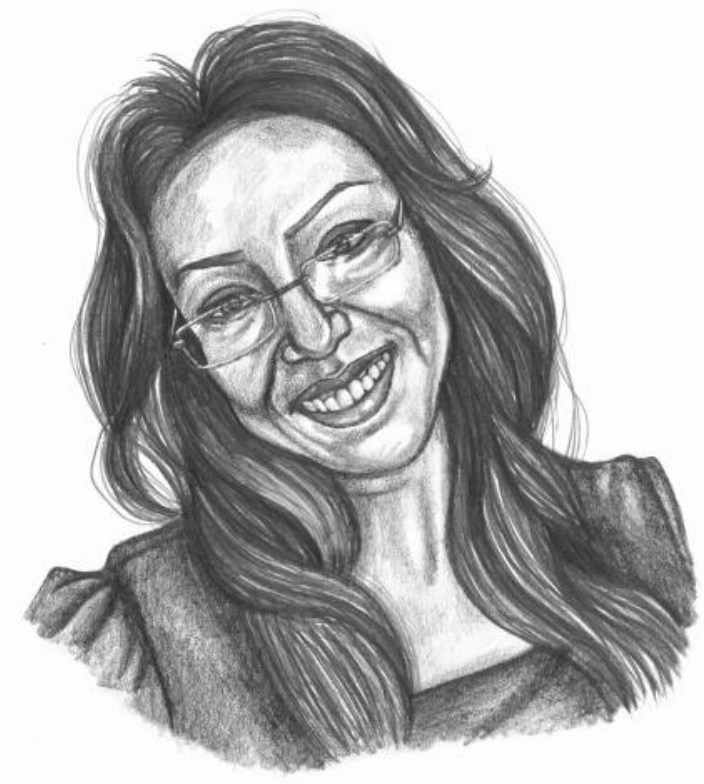


The Syrian crisis is entering its seventh year, and civilians continue to suffer and bear intolerable unique social consequences resulted of the most aggressive violent phenomena after the Second World War. The Humanitarian Needs Overview (HNO) for 2017 estimates that 6.3 million people are displaced within Syria and more than 6 million are outside the country. The assumptions of the humanitarian response have concluded a total number of 13.5 million people require humanitarian assistance and protection (UN OCHA, 2016).

General conditions for Syrians are varied according to their physical location and availability of humanitarian support through national actors. Sanctions and fuel shortages, combined with unemployment, price rises, and inflation have adversely impacted the purchasing power of the communities. Within Syria, access to health care services is a discussable issue according to location. In some areas, health care facilities have become military targets and people are afraid to seek health care. At least 1 in 4 public hospitals in the country is not functional, and those in still in service are overwhelmed with patients. The dysfunction of health services is compounded by shortages of medical supplies, equipment, and medications throughout the country. A number of Syrian medical staff have mobilized to set-up informal health care facilities to provide life-saving surgeries, stabilization, first aid treatment, and primary health care services for those unable to access other health services. However, the majority of senior and most qualified medical staff have fled the country due to the crisis, while those staffing informal health facilities are in dangerous environments near active conflict and are frequently targeted.

The humanitarian actors have done serious efforts to enable streamlined gender and protection principles in field assessments and program designs of health, NFI, protection, WASH and nutrition sector activities. To inform interventions, actors were keen to design interventions which assessed single-sex and mixed groups and interviewed caregivers to identify protection concerns of boys and girls of various ages. Meeting the needs of vulnerable groups is integrated into training on psychosocial support, and GBV. Actors who work with camp management and relief committees have reported their abilities to identify vulnerable groups including the elderly and people with disabilities to create links to complementary services. Nevertheless, interventions and programs related to mental health disorders, traumas, and psychological affection have not been reported frequently.

The operational definition of Mental Health and Psychosocial Support (MHPSS) is a composite term used to describe any type of support that aims to protect or promote psychosocial well-being and/or prevent or treat mental distress. Interventions towards achieving that target include psychological first aid and linking survivors with other services, psychosocial interventions (such as groups activities), and, where indicated, specialist mental health care. It also includes engaging the broader community support to potentially play a role in protecting the dignity, promoting psychosocial wellbeing and preventing mental health problems associated with gender-based violence and the stigmatization/isolation of the survivor. Not all survivors have the same psychological and social needs following SGBV incidents. 
Not all survivors of Gender-based violence will need or want emotional support, counseling or help, nevertheless, access to psychosocial support should always be available. The cornerstone of social work is trust. Like for health care services providers, trust is required to disclose such sensitive issues, and as medical care, social work is to be considered a specialized treatment as well. Consequently, as for medical profession, social workers are also bound by a professional obligation to client/patient confidentiality.

Situations of health care and protection support is different for the those outside Syria where we are looking at a number of more than 6 million refugees who are scattered between different types of accommodations in five countries: Iraq, Lebanon, Jordan, Turkey, and Egypt.

Mental health needs, as well as protection and other types of needs, have differed and changed frequently between locations. A number of factors are affecting the humanitarian response plans to meet the gaps in service provision to survivors of mental health illnesses like hosting country stand on the refugee status of Syrians in their countries, existing health system capacities and integration/acceptance by the citizens of those countries for Syrian refugees.

The paper Syrian Refugee Children and Mental Health Trauma by Kathleen O'Brien has reflected a very specific perfect drawing of the mental health status of children in Zaatari refugee camp in comparison to other countries where refugees have extended to reach specifically in Canada. A well-researched paper with good references, and based on an excellent analysis and review of available pieces of literature on Syria crisis.

Dr. Alahmer is a senior reproductive health and Gender Based violence professional, a member of the international association of facilitators having experience of more than 16 years, and a certified trainer of trainers for Reproductive health, GBV, HIV, Obstetric emergencies, monitoring and evaluation, Project cycle management, epidemiology, clinical Management of Rape survivors, EMOC, MISP, HIV peer leadership and PEP Provision and Counseling, PSEA, case management and psychosocial counselling. Holding two medical specialties, Dr. Alahmer is an obstetrician/gynecologist and public health professional, in addition to her International Diploma of Humanitarian Assistance from Fordham University in New York, USA. She started her humanitarian career at the time of the crisis in Darfur, where she worked as a medical coordinator for a Sudanese Medical NGO between 2004 and 2005. She moved to MSF where she served as Medical Activity Manager in 2007. In 2008 she joined UNFPA as Officer in Charge (OiC), RH and GBV officer for Zalingie sub office., One year later she joined the United Nations Mission in Sudan, where she served as trainer of trainers and counselor for the HIVIAIDS advisory. Early in 2011 with the escalation of Libya revolution, Dr. Alahmer joined the International Medical Corps Organization (IMC) Libya team as GBV program manager and moved on to Turkey with the same responsibilities serving the Syrian refugees for another one year. Since 2014 she has been a GBV Program specialist in Damascus dealing on daily bases with the Syria crisis from within the country.

\section{BIBLIOGRAPHY}

2017 Humanitarian Needs Overview: Syrian Arab Republic. UN OCHA, December 2016. Retrieved from https:/www.humanitarianresponse.info/system/files/documents/ files/2017_syria_hno_2.pdf. 